\title{
Impact of Pulsar Giant Pulses on Distant Clocks Comparison
}

\author{
Yury P. Ilyasov, ${ }^{1}$ Vladimir E. Zharov ${ }^{2}$ and M. Sekido ${ }^{3}$ \\ ${ }^{1}$ Pushchino Radio Astronomical Observatory of P.N. Lebedev Physical Institute of the Russian \\ academy of science, Pushchino, Moscow region, Russia \\ email: ilyasov@prao.ru \\ ${ }^{2}$ Department of Physics, Moscow State University, Moscow, Russia \\ email: zharov@sai.msu.ru \\ ${ }^{3}$ National Institute of Information and Communications Technology \\ Kashima, Japan \\ email: sekido@nict.go.jp
}

\begin{abstract}
New method of precise clocks comparison based on observation and registration of giant pulses of the millisecond pulsars is discussed. It was shown that expected accuracy of comparison is about $0.2-2 \mathrm{~ns}$ and depends on uncertainty of delay in the Earth ionosphere and troposphere.
\end{abstract}

Keywords. Pulsars, time transfer, giant pulses

Now it is well-known that several pulsars occasionally generate several times per hour huge superfine short pulses, which flux density is about million times more than an average level. The most famous pulsars, having such signatures, are the millisecond pulsar B0531+21, relatively young pulsar in the Crab nebulae, and the other such as $\mathrm{J} 0218+4232$, J1939+2134, J1959+2048. Their radio emission features are studied now in detail but real mechanism of such huge generation power is not well understood yet (Hankins \& Eilek 2007, Popov et al. 2008). Giant pulses (GP) being as shorter as part of nanosecond and received several times per hour could be used for distant clocks precise comparison (Ilyasov et al. 1991, Ilyasov et al. 2007). Now VLBI technique is under the study to apply for time transfer within accuracy about $0.01-0.1 \mathrm{~ns}$. But it can be done after correlation procedure of VLBI data, which takes several hours or even days. A new method of distant clock comparison could be done promptly only after communication between clock stations in the first step without any correlation procedure. When GP is about one ns duration a precision time transfer could be comparable with VLBI technique. The fast wide bandwidth facilities should be used to detect GP. In this new method the same limiting accuracy factors of VLBI should be taken into consideration. Depending on frequency of observations clock comparison can be done with accuracy of about $0.2-2$ ns. This method is much more cheap than Two-Way Satellite Time Transfer (TWSTT) and can be used not only for ground-base clocks but for the clocks in space.

This work was supported by the RFBR grants 06-02-16816, 09-02-00922.

\section{References}

Hankins, T. H. \& Eilek, J. A. 2007, ApJ, 670, 693

Popov, M., Soglasnov, A., et al. 2008, J.Astr, 85, 1

Ilyasov, Yu., Kuzmin, A., et al. 1991, Technique of clocks synchronization by pulsars signals, Patent (Soviet Union) No 1669301

Ilyasov, Yu, Oreshko, V., et al. 2007, Proc. of the IAA RAS (St. Petersburg), 17, 128 